\title{
A Real-Time 3D Reconstruction System for Screening Icosahedral Particles Under Different Conditions at the Microscope.
}

\author{
Giovanni Cardone ${ }^{1}$, Xiaodong Yan¹, Robert S. Sinkovits² and Timothy S. Baker ${ }^{1,3}$ \\ 1. Department of Chemistry and Biochemistry, University of California San Diego, La Jolla, CA 92093 \\ 2. San Diego Supercomputer Center, University of California San Diego, La Jolla, CA 92093 \\ 3. Division of Biological Sciences, University of California San Diego, La Jolla, CA 92093
}

Automated data collection systems [1] allow one to easily acquire large numbers of images that can be then processed by single-particle techniques to generate three-dimensional (3D) maps. However, the determination of the best sample conditions for such high-throughput processing can still require lengthy and manual work. In some cases it is necessary to screen different sample preparations to find the one giving the physiological state of interest, whereas in others screening can be used to optimize buffer conditions. This analysis can be facilitated by the use of multi-specimen holders that permit loading more than one grid in the microscope, or by novel systems under development that dispense multiple samples onto a single grid [2]. Still, the evaluation of each condition is based on the analysis of 2D images, which provide only partial information on the properties of the specimen. As a solution for the analysis of particles with icosahedral symmetry, we are developing a real-time alignment and reconstruction scheme that provides a $3 \mathrm{D}$ volume from a single image acquired at the microscope under cryogenic and low dose conditions.

Our new system reads images as they are acquired at the microscope and processes them independently on a small computer cluster, making use of up to 20 processors in parallel. The implementation provides a graphical user interface for monitoring the results, and it relies on the package AUTO3DEM [3] for all processing steps. The only input parameter required by the user is an approximate radius of the particle being examined. Each micrograph is initially binned to speed up computations. Defocus estimation and particle picking are then performed in parallel. Finally, the extracted particle images are used to estimate a 3D density map, without using any reference model. Following the Random Model Method [4], ten initial models are generated using random orientations, and they are refined independently for ten iterations, with each refinement computed on 2 processors. All steps are performed without the need for user intervention.

We have tested the performance of our automated system on several existing datasets, chosen as representative of different acquisition conditions. In most instances, the 3D reconstruction obtained from a single micrograph can be supplied to the microscopist in a time frame of between 30 seconds and 2 minutes, which mostly depends on the pixel diameter of the particles. The probability of obtaining a reliable reconstruction from one micrograph is primarily determined by the quality of the sample. Success rates close to $100 \%$ are realized when sample conditions are optimal, and decrease to $\sim 60 \%$ when contaminants are present or the ice is too thick. Test results on a baboon reovirus (BRV) dataset [5] illustrate (Fig. 1) an example in which our new system can discriminate particles with different conformations. In this dataset, a portion of the virus' core turret protein (the "flap”) assumes one of two distinct conformations on separate microscope grids. Such variability cannot be detected from the raw micrographs directly but is evident when 3D reconstructions from single micrographs acquired from different grids are compared. Each reconstruction was obtained in less than two minutes. 
The proposed approach is well-suited for providing rapid feedback of 3D information to the microscopist during the screening of multiple samples loaded in a microscope. Based on many test results including those with BRV, our system has proven to be capable of assessing small structural differences between samples that could not be detected by simple visual analysis of the acquired images. Real-time feedback to the microscopist not only helps with screening sample properties, but also permits rapid evaluation of microscope conditions and provides an initial template for high-throughput, automated data collection. In addition, it can potentially be used to study dynamic events when combined with in-situ electron microscopy techniques [6,7].

\section{References:}

[1] C Suloway et al., J Struct Biol 151 (2005), p. 41.

[2] T Jain et al., J Struct Biol 179 (2012), p. 68.

[3] X Yan, RS Sinkovits and TS Baker, J Struct Biol 157 (2007), p. 73.

[4] X Yan et al., J Struct Biol 157 (2007), p. 211.

[5] X Yan et al., J Virol 85 (2011), p. 7483.

[6] BL Gilmore et al., Lab Chip 13 (2013), p. 216.

[7] This work is supported by NIH grant GM087708 to T.S.B.
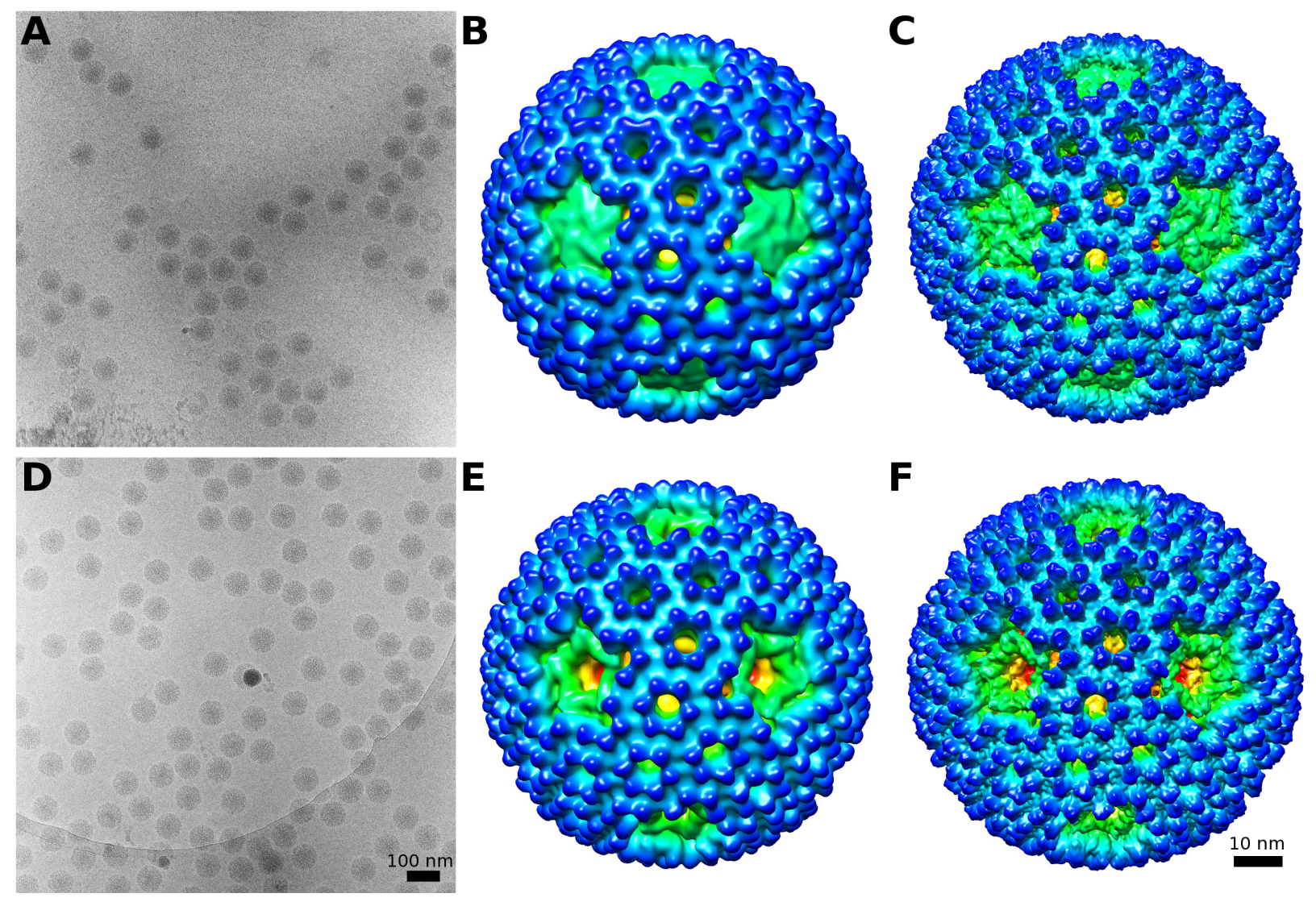

Figure 1. Baboon reovirus, in presence (top row) and absence (bottom row) of flap density around the five-fold vertices. (A,D) micrographs acquired at $300 \mathrm{kV}$, dose $\sim 25 \mathrm{e}^{-} / \AA^{2}$, defocus $\sim 3 \mu \mathrm{m}$. (B,E) 3D reconstructions using 36 and 77 particles automatically extracted from micrographs shown in A and D. Total processing time for the reconstruction on $20 \mathrm{cpu}$ cores was 85 and $118 \mathrm{~s}$, respectively. (C,F) for comparison, 3D reconstructions from 2138 and 2993 particles selected from the full dataset [5]. 